\title{
Telomere length is highly heritable and independent of growth rate manipulated by temperature in field crickets
}

\author{
Jelle Boonekamp ${ }^{1,2}$ (๑) | Rolando Rodríguez-Muñoz ${ }^{1}([$ |

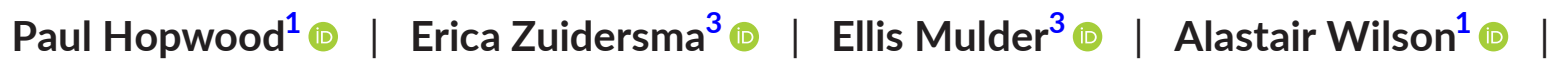 \\ Simon Verhulst ${ }^{3}$ (i) | Tom Tregenza ${ }^{1}$ ()
}

${ }^{1}$ Centre for Ecology \& Conservation, School of Biosciences, University of Exeter, Exeter, UK

${ }^{2}$ Institute of Biodiversity, Animal Health \& Comparative Medicine, College of Medical, Veterinary \& Life Sciences, University of Glasgow, Glasgow, UK

${ }^{3}$ Groningen Institute for Evolutionary Life Sciences, University of Groningen, Groningen, The Netherlands

\section{Correspondence}

Jelle Boonekamp, Centre for Ecology $\&$ Conservation, School of Biosciences, University of Exeter, Penryn Campus, TR10 9FE, UK.

Email: jjboonekamp@gmail.com

Funding information

Natural Environment Research Council (NERC), Grant/Award Number: NE/ R000328/1; Marie Skłodowska-Curie, Grant/Award Number: 792215

\begin{abstract}
Many organisms are capable of growing faster than they do. Restrained growth rate has functionally been explained by negative effects on lifespan of accelerated growth. However, the underlying mechanisms remain elusive. Telomere attrition has been proposed as a causal agent and has been mostly studied in endothermic vertebrates. We established that telomeres exist as chromosomal-ends in a model insect, the field cricket Gryllus campestris, using terminal restriction fragment and Bal 31 methods. Telomeres comprised TTAGGn repeats of $38 \mathrm{~kb}$ on average, more than four times longer than the telomeres of human infants. $\mathrm{Bal} 31$ assays confirmed that telomeric repeats were located at the chromosome-ends. We tested whether rapid growth between day 1 , day 65 , day 85 , and day 125 is achieved at the expense of telomere length by comparing nymphs reared at $23^{\circ} \mathrm{C}$ with their siblings reared at $28^{\circ} \mathrm{C}$, which grew three times faster in the initial 65 days. Surprisingly, neither temperature treatment nor age affected average telomere length. Concomitantly, the broad sense heritability of telomere length was remarkably high at $100 \%$. Despite high heritability, the evolvability (a mean-standardized measure of genetic variance) was low relative to that of body mass. We discuss our findings in the context of telomere evolution. Some important features of vertebrate telomere biology are evident in an insect species dating back to the Triassic. The apparent lack of an effect of growth rate on telomere length is puzzling, suggesting strong telomere length maintenance during the growth phase. Whether such maintenance of telomere length is adaptive remains elusive and requires further study investigating the links with fitness in the wild.
\end{abstract}

KEYWORDS

field cricket, Gryllus campestris, telomere

\section{1 | INTRODUCTION}

Genetic factors and early life developmental conditions affect growth, with long-term consequences for adult health and fitness, but the underlying proximate mechanisms remain elusive (Dmitriew, 2011). Biomarker variables could shed light on causal mechanisms, in particular when manipulated growth rate is reflected in the biomarker and when the biomarker variable in turn is predictive of 
fitness (Boonekamp et al., 2017; Frangakis \& Rubin, 2002). Telomere length is advocated as a useful biomarker in this context, for example Monaghan and Ozanne (2018).

Telomeres are highly conserved nucleoprotein structures that form protective caps at the chromosome-ends where they prevent catastrophic end-to-end chromosome fusion (Armanios \& Blackburn, 2012). Vertebrate telomeres comprise tandem repeats of a 5'-TTAGGG-3'sequence protected by shelterin proteins (Blackburn, 1991). At the chromosome level, telomeres shorten with each cell division through incomplete DNA replication (Olovnikov, 1973), single strand breaks induced by reactive oxygen species molecules (Zglinicki, 2002), and loss of the single strand overhang (Stewart et al., 2003). Telomeres may be restored through the action of telomerase, but the activity of this enzyme rapidly diminishes during early development and it is thought to be mainly inactive in somatic tissues (Hug \& Lingner, 2006). Consequently, telomeres shorten with age, with the fastest telomere erosion observed during growth (Benetos et al., 2019; Salomons et al., 2009; Zeichner et al.,1999). In adult humans, telomere shortening is related to stress (Entringer et al., 2011; Epel et al., 2004), disease (Calado \& Young, 2009), and lifestyle factors (Lin et al., 2012) and telomere length predicts mortality risk (Boonekamp et al., 2013). Telomere length also predicts mortality (Wilbourn et al., 2018) and fitness (Eastwood et al., 2019) in natural populations of vertebrates and the rate of telomere attrition is negatively correlated to maximum lifespan across species (Tricola et al., 2018). Gradual telomere erosion with growth and/ or age could reflect a multilevel continuous process of cell division, damage, and repair, linked to fitness. It is therefore intuitive to consider telomere length at the end of the growth period and/or the rate of telomere attrition during growth as candidate biomarkers linking growth to fitness, a supposition supported by earlier studies, for example, Heidinger et al., (2012) and Boonekamp et al., (2014).

The proximate mechanism of telomere length as a biomarker of the cost of growth is based on the premise that growth acceleration increases the number of telomere base pairs that are lost per cell division (Monaghan \& Ozanne, 2018). Dependence of the rate of telomere attrition on growth rate could for example arise by increased oxidative stress associated with growth acceleration (Alonso-Alvarez et al., 2007; Smith et al., 2016), but see Chatelain et al., (2019). However, because both the total number of cell divisions and the rate at which cells divide may contribute to telomere loss, experiments are required that disentangle the effects of size and growth rate on telomere length. Only a few studies have attempted to disentangle the effects of growth rate and size on telomere length and they show mixed results: In the common tern (Sterna hirundo), incubator temperature affected the time until hatching without affecting size at hatching, and faster growth corresponded with reduced telomere length of hatchlings (Vedder et al., 2018). A comparable experimental study showed similar effects of temperature on growth rate and telomere attrition in quail hatchlings (Stier et al., 2020). In contrast, a similar experiment in Atlantic salmon larvae found no association between growth rate and telomere length
(McLennan et al., 2018). We are unaware of other experimental studies attempting to distinguish the effects of size and growth rate on telomere length. Hence whether growth acceleration reduces telomere length remains unclear.

The relationship between telomere dynamics and fitness has been studied almost exclusively in vertebrates and whether similar patterns exist in invertebrates remains elusive, but see Jemielity et al., (2007). Terminal telomeres appear to be ubiquitous in metazoans, with the exception of dipterans (Gomes et al., 2010). In insects with terminal telomeres the predominant telomeric sequence is 5'-TTAGG-3', which is only one guanine nucleotide short of the vertebrate sequence, and many insects appear to express telomerase during development (Korandová et al., 2014). Furthermore, fluorescence in situ hybridization (FISH) reveals that insect TTAGG repeats occur almost exclusively at the chromosome ends (Frydrychová et al., 2004; Okazaki et al.,1993; Vítková et al., 2005), suggesting that insects are virtually free from interstitial telomeric repeats. These findings raise the question of whether telomere length is a biomarker of growth and fitness in insects. If confirmed, telomere length could be used as study endpoint, eliminating the need to longitudinally monitor individual insects which are notoriously difficult to follow in the wild.

As ectotherms, insects have enormous developmental plasticity and as such are powerful systems for studying the effects of developmental conditions on growth and fitness (Richard et al., 2019). Growth rate can be manipulated through modification of temperature in ectotherms (Gilbert \& Raworth, 1996), and because environmental temperatures are variable it can be assumed that insects show an evolved response to temperature variation. Temperature is expected to have differing effects in ectotherms versus endotherms, for example, because body temperature of ectotherms depends to a larger degree on behavioural adjustments. Endotherms have evolved to operate at a narrow range of body temperatures, so attempts to manipulate these are likely to cause acute stress. Ectotherms have evolved to tolerate varying temperatures, and are not expected to attempt to compensate, for instance by increasing food consumption and metabolic rate, in the same magnitude as endotherms. However, until a critical upper limit is reached, temperature inevitably increases metabolic rate, increasing both oxygen consumption (Roe et al.,1980) and oxidative stress in insects (Das et al., 2018; Lalouette et al., 2011) and we therefore predict that accelerated growth through temperature will incur a physiological cost in insects, potentially resulting in shorter telomeres.

Here, we use nymphs of the field cricket Gryllus campestris to study the effects of growth manipulated through temperature on telomere length. Previous FISH studies show that telomeres exist as chromosome-ends in two related cricket species (G. assimilis and Eneoptera surinamensis) (Palacios-Gimenez et al., 2015). We verified that there are telomeric repeats at the chromosome-ends in $G$. campestris nymphs, using a combination of approaches (see methods). Subsequently, we collected wild G. campestris from high and low altitudes in Asturias, Spain and exposed their offspring nymphs 
to high and low temperature conditions using a full factorial design. Based on personal observations, which we later verified to be true (Tregenza et al., submitted), we anticipated altitudinal variation in growth rate in response to temperature. Here, we aimed to study the effects of temperature and age on telomere length against the background of the naturally occurring altitudinal variation in growth rate (see methods and Figure 1 for an overview of the experimental design).

We predicted that growth acceleration by temperature results in progressively shorter telomeres with longer exposure to higher temperature. However, our main findings indicate that neither temperature, growth, age, nor altitude affected telomere length, whereas, unexpectedly, a large portion of the among individual variation appeared to be explained by sibling relatedness. Our statistical analyses of this striking finding prompted additional post hoc quantitative genetic analysis using an "animal model" to test whether similarity by relatedness is explained by additive genetic effects. However, since we did not design our study from the outset to conduct quantitative genetic analyses, there are some caveats in the interpretation of these analyses, which limit the confidence we have in our estimates of additive genetic variance and narrow sense heritability of telomere length.

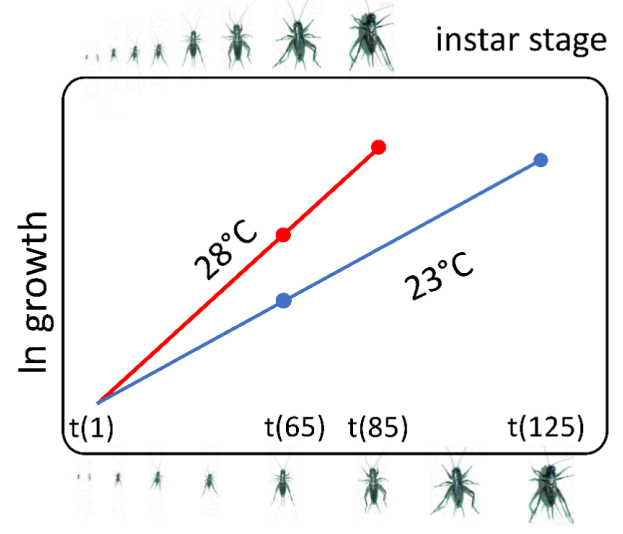

FIGURE 1 Schematic overview of the experimental design. Early stage nymphs entered temperature treatment conditions at $\mathrm{t}(1)$. Temperature conditions $\left(28^{\circ} \mathrm{C} \& 23^{\circ} \mathrm{C}\right)$ were replicated using a duplicate climate room setup. For each family, siblings were divided over the four climate rooms such that each family was represented in each room by at least one sibling. Dots on the temperature lines represent the moment nymphs were snap-frozen/sampled. Half of the nymphs in both temperature conditions were weighed and snap-frozen after 65 days of treatment. This group represents same age, different size siblings. The second half of the $23^{\circ} \mathrm{C}$ group were sacrificed after 125 days of treatment with the aim of achieving the same size relative to $28^{\circ} \mathrm{C}$, but different chronological age siblings. Because these two groups of 65 days @ $28^{\circ} \mathrm{C}$ and the 125 days @ $23^{\circ} \mathrm{C}$ groups differ in two dimensions, both in time and temperature, we included a third group of $28^{\circ} \mathrm{C}$ that was sacrificed at an intermediate age (t85), thus achieving greater statistical power to disentangle the effects of temperature from the effects of age

\section{2 | MATERIALS AND METHODS}

\section{1 | Cricket collection and breeding}

Gryllus campestris has a relatively large genome comprising $2 n=0^{*}$ 29 (Türkoğlu et al., 2003). A genome scan (unpublished, summary available from Tregenza) indicates that the genome size is $2.5 \mathrm{~Gb}$ and includes a high level of repeats. We collected adult and subadult G. campestris crickets from 10 sites in Northern Spain (Table 1) in late April and early May 2018 using burrow traps (for details on trapping see Rodríguez-Muñoz, Boonekamp, Liu, Skicko, Fisher, et al., 2019). Five sites were at low altitude (84 $\pm 59 \mathrm{~m}$ above sea level [mean \pm SD]) and five sites were at high altitude $(1148 \pm 164 \mathrm{~m})$. Captured crickets were maintained in a research facility in the study area (near location L3, see Table 1), in individual $7 \times 7 \times 10 \mathrm{~cm}(\mathrm{~L} \times \mathrm{W} \times \mathrm{H})$ metal mesh cages (pen holders) in a single temperature-controlled room at $25^{\circ} \mathrm{C}$ with a 07:0022:00 $\mathrm{h}$ light cycle, reflecting the day length at their source locations. The position of the boxes was rearranged at least weekly to ensure that potential temperature gradients within the room could not systematically affect populations. Once individual females had been adult for at least three days they were mated to a single male from their own population by placing the pair together in a $9 \mathrm{~cm}$ circular plastic container and observing them until mating occurred, typically within an hour $(n=59)$. In a further seven cases the pair failed to mate within $1 \mathrm{~h}$, and the male was replaced with a second male with whom she mated within the next 2-3 days. After mating, females were provided with a $3 \mathrm{~cm}$ petri dish with wet sand for egg laying. Dishes were checked and eggs removed every other day. Eggs were transferred to a new petri dish containing cotton wool moistened with water and maintained at $25^{\circ} \mathrm{C}$ in an incubator (1-3 petri dishes per female). Females typically laid between a few tens to a few hundreds of eggs each, which were spread out evenly on the surface of the cotton wool to reduce the potential for mould to spread. As nymphs hatched over a period of $\sim 8$ days, they were transferred to $7 \times 7 \times 6 \mathrm{~cm}$ boxes and sent by air to the University of Groningen (the Netherlands).

TAB LE 1 Sampling locations in Northern Spain with altitude in meters

\begin{tabular}{|lrl|}
\hline Code & Altitude & Coordinates \\
\hline L1 & 50 & N43 33.258 W6 51.692 \\
\hline L2 & 130 & N43 22.473 W6 09.192 \\
\hline L3 & 20 & N43 31.610 W5 37.590 \\
\hline L4 & 58 & N43 27.095 W4 55.195 \\
\hline L5 & 160 & N43 17.094 W4 26.388 \\
\hline H6 & 1260 & N43 02.099 W6 27.285 \\
\hline H7 & 1300 & N43 04.106 W6 11.156 \\
\hline H8 & 1160 & N43 09.686 W5 54.583 \\
\hline H9 & 880 & N43 16.514 W5 30.243 \\
\hline H10 & 1140 & N43 06.309 W5 13.327 \\
\hline
\end{tabular}




\subsection{Temperature treatment}

At the University of Groningen, we housed the nymphs in four identical climate rooms to control temperature, humidity, and the daily light cycle. We set the temperature of two of the four rooms to $28^{\circ} \mathrm{C}$ and the other two rooms to $23^{\circ} \mathrm{C}$, whilst relative humidity was maintained at 50\%. All four rooms were on a $12 \mathrm{~h}$ light: dark cycle. We took a median of 20 nymphs from each dam (range 6-15) and divided siblings over the four climate rooms such that each room had representatives of each family. To minimize potential negative effects of density, up to 10 siblings (median $\pm S D=6 \pm 4.4$ ) were kept in single boxes of $10 \times 10 \times 6 \mathrm{~cm}$; we used multiple boxes for large families.

We snap-froze nymphs in liquid nitrogen at three different time-points to create "same chronological age" nymphs that differed in their body size due to the temperature treatment, as well as "same size but different chronological age" nymphs. Nymphs that were sharing the same box were frozen simultaneously. The freezing of the three batches was timed as follows: We sacrificed half of all the boxes (randomly preselected) of nymphs in both treatment groups after 65 days of treatment. The second half of the $28^{\circ} \mathrm{C}$ group was sacrificed after 85 days of treatment, and the second half of the $23^{\circ} \mathrm{C}$ group after 125 days, with the intention that these sample groups had grown to approximate the same size. This design allowed us to disentangle the effects of age and temperature on body mass (see Figure 1 for a schematic overview) We measured body mass immediately before snap-freezing and nymphs were stored at $-80^{\circ} \mathrm{C}$ until DNA extraction for telomere length measurement. In total 545 nymphs from 59 dams survived to sampling, with $n=188$ (day $65 @ 23^{\circ} \mathrm{C}$ ); $n=195$ (day $65 @ 28^{\circ} \mathrm{C}$ ); $n=53$ (day $125 @ 23^{\circ} \mathrm{C}$ ); and $n=109$ (day $85 @ 28^{\circ} \mathrm{C}$ ). Because TRF measurements are laborious, our limited capacity prevented us from measuring all 545 individuals, hence we measured telomere length in a subset of 77 randomly selected nymphs from 29 dams. Nymphs were selected at random, per treatment per female when available, but with post-selection verification that the bodymass distributions were unbiased (see Figure S1).

\section{3 | DNA extraction}

DNA was extracted for telomere length measurements. Up to $100 \mathrm{mg}$ of homogenized whole-thorax tissue was obtained from frozen specimens and prepared for DNA-extraction using the Blood \& Cell Culture DNA Midi Kit (\#13343) by Qiagen, using 100/G Genomic-tips, following the manufacturer's protocol described in the Qiagen Genomic DNA Handbook. DNA concentrations were measured in duplo with a Nanodrop 2000c from Thermo. Solutions were scaled to a allow a maximum of $4 \mu \mathrm{g}$ genomic DNA to fit homogeneously into a $100 \mu \mathrm{l}$ agarose plug, using the CHEF Genomic DNA Plug Kit (\#170-3591) by Bio-Rad Laboratories. Only one quarter of each agarose plug was used for telomere length analysis.

\section{4 | Nuclease Bal 31 assay}

We carried out a Bal 31 assay on six individual adult field crickets that were not part of the temperature experiment in order to establish that telomeric repeats occurred at the chromosome-ends. This assay rests on the premise that $\mathrm{Bal} 31$ degrades DNA from the chromosomal ends and hence if $\mathrm{Bal} 31$ exposure reduces telomere length then this is demonstrative of their end-cap position (Atema et al., 2019). A positive result however does not rule out the coexistence of interstitial telomeric repeats, which can potentially be substantial in number and highly variable among individuals. The outcome of the $\mathrm{Bal} 31$ assay is therefore particularly informative when the assay is done using denatured DNA: long interstitial telomeric repeats will then be revealed by the telomere probe binding to all TTAGG sequences on the genome, including interstitial repeats (rather than the probe's affinity being restricted to binding to the single strand telomere overhang of intact double stranded DNA). We therefore carried out the Bal 31 assay and denatured DNA after gel electrophoresis (see Section 2.5 below). Bal 31 digestion was performed in agarose plugs containing isolated genomic DNA. Prior to the digestion, plugs were incubated for $1 \mathrm{~h}$ with the $\mathrm{Bal} 31 \mathrm{1} \times$ reaction buffer (supplied by the manufacturer). Each agarose plug was cut into four equal pieces in separate reaction tubes. One piece $(T=0)$ was directly washed on ice $3 \times$ for 10 min with 50 mM EDTA followed by $3 \times$ for $10 \mathrm{~min}$ with $20 \mathrm{mM}$ TRIS. The other three pieces were incubated for $20 \mathrm{~min}(T=20), 80 \mathrm{~min}(T=80)$ and $240 \mathrm{~min}(T=240)$ respectively, with $200 \mu \mathrm{l} 0.1 \mathrm{U}$ Nuclease Bal 31 enzyme (New England Biolabs \#M0213S) at $30^{\circ} \mathrm{C}$, immediately followed by washing similar to $T=0$ to stop the digestion. After digestion, the TRF-protocol as described below was performed to measure the molecular size of TTAGG fragments.

\subsection{Terminal restriction fragments protocol}

We measured telomere length using similar procedures as in Boonekamp et al., (2014). Briefly, agarose plugs were preincubated for $1 \mathrm{~h}$ with $1 \times$ Cutsmart buffer (New England Biolabs), followed by overnight incubation with $20 \mathrm{U}$ restriction enzymes Hinfl and $20 \mathrm{U}$ Rsal (New England Biolabs) at $37^{\circ} \mathrm{C}$. Telomeric repeats of sequence TTAGG were separated from restricted DNA with Pulsed Field Gel Electrophoresis (CHEF-DR II system by BioRad) in a $0.8 \%$ agarose gel. Electrophoresis settings were: $4.8 \mathrm{~V} /$ $\mathrm{cm}, 1-25 \mathrm{~s}$ switch time, $22 \mathrm{~h}$ at $14^{\circ} \mathrm{C}$ and for the $\mathrm{Bal} 31$ assay we used: $3.5 \mathrm{~V} / \mathrm{cm}, 0.5-7 \mathrm{~s}$ switch time, $24 \mathrm{~h}$ at $14^{\circ} \mathrm{C}$. Each gel also contained ${ }^{32} \mathrm{P}$-end-labeled size ladders (Molecular Weigh Marker $\mathrm{XV}$, by Roche Diagnostics and $1 \mathrm{~Kb}$ DNA ladder by New England Biolabs). After electrophoresis, the gels were fixed, dried and denatured. Denaturation was performed by incubating the dried gel with denaturation buffer (1.5 M NaCl, 0.5 M NaOH) 3 times for $30 \mathrm{~min}$ at room temperature, with gentle agitation, followed by neutralization buffer (0.5 M Tris- $\mathrm{HCl}$ pH 8.0, 1.5 M NaCl) twice for $30 \mathrm{~min}$ at room temperature, with gentle agitation. Hybridization 
of telomeric repeats occurred overnight at $37^{\circ} \mathrm{C}$ with a ${ }^{32} \mathrm{P}$-endlabeled oligonucleotide $\left(5^{\prime} \text {-CCTAA- } 3^{\prime}\right)_{4}$ with gentle agitation in hybridization buffer (0.5 M Na2HPO4, 7\% SDS, 1 mM EDTA). After washing away unbound oligonucleotides, the gel was exposed overnight to a phosphor screen (Perkin Elmer) and read at $600 \mathrm{dpi}$ with the Cyclone Storage Phosphor System by Perkin Elmer.

\subsection{Statistical analyses}

We analysed the effects of treatment on body mass and telomere length using mixed effects models with family identity (ID) as random effect. We corrected for gel differences in our telomere length analyses by including gel-ID as a fixed effect (4 gels). We subsequently analysed temperature and age as fixed effects (ordinal factors) and the intercept therefore reflects the dependent variable at $23^{\circ} \mathrm{C}$ and at day 65 . Mixed effects models were performed with the ASreml4 package (Butler et al., 2018) in R (R Core Team, 2020) using restricted maximum loglikelihood estimation. We used F-tests and log-likelihood ratio tests to determine the statistical significance of fixed and random effects, respectively.

We calculated the coefficient of variation of telomere length by dividing the square root of the residual variance $\left(V_{R}\right)$ of a model that only included gel-ID as a fixed effect with the mean telomere length $(\bar{x}): C V(x)=\sqrt{V_{R}} / \bar{x}$. This is an appropriate estimate of biological variation because it corrects for methodologically induced variation and then scales the residual biological variation to the mean telomere length. We calculated the broad sense heritability $\left(\mathrm{H}^{2}\right)$ of telomere length following Falconer and Mackay (1996) by dividing the between family variance $\left(V_{f}\right)$ estimated by the family ID random effect, with the total variation and then multiplying by two (since full siblings are on average related by 0.5$): H^{2}=2 \cdot V_{f} /\left(V_{f}+V_{R}\right)$.

Our statistical analyses prompted additional post hoc quantitative genetic statistical analyses (see Results \& Discussion). Briefly, we conducted these additional quantitative genetic analyses with ASreml4 in R by fitting a pedigree random effect based on the fullsibling relatedness. Narrow sense heritability was estimated by scaling the additive genetic variance (estimated via the pedigree random effect) to the residual variance: $h^{2}=V_{A} /\left(V_{A}+V_{R}\right)$. The $95 \%$ heritability confidence intervals were obtained by multiplying the standard errors by 1.96 , and the standard errors were estimated using the ASreml vpredict function.

\section{3 | RESULTS}

\section{1 | Descriptive results}

Bal 31 reduced telomere length in all six individuals tested for this purpose (Figure 2). We found no evidence for the presence of interstitial telomeric repeats as revealed by the absence of a banded pattern with single stranded DNA (Figure 2). This method only reveals large interstitial telomeric repeats, but our findings are in

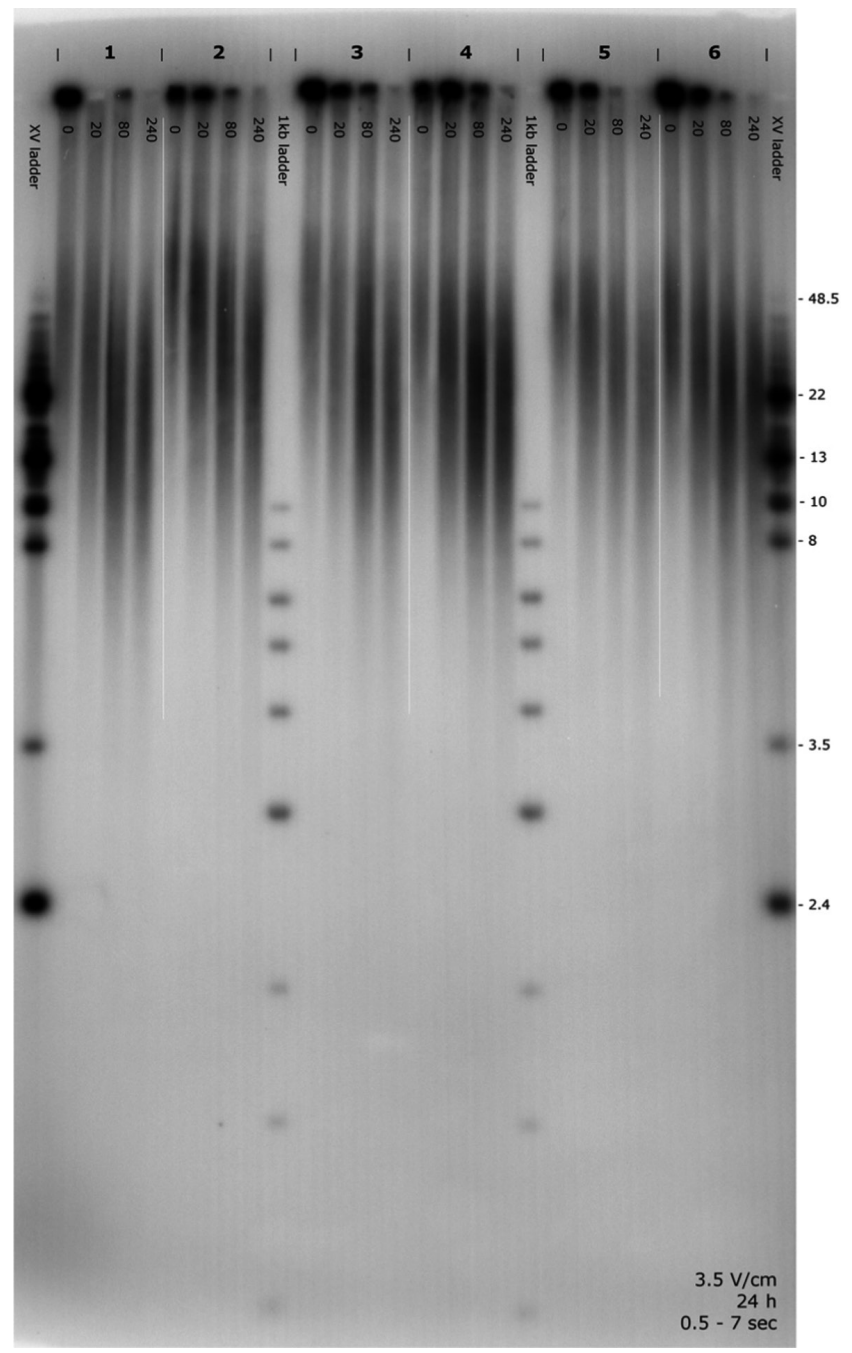

FIGURE 2 Pulsed field gel electrophoresis showing TTAGG $_{n}$ telomere restriction fragments from the denatured chromosomes of six individual crickets (indicated by the numbers 1-6). XV molecular size ladders are shown on the outer lanes and shorter $1 \mathrm{~kb}$ ladders are shown between crickets 2-3 and 4-5. The photo shows four aliquots of DNA sample per cricket and these aliquots were exposed to 0 (i.e., untreated), 20, 80, and 240 min of Bal 31. Each time series clearly shows that $\mathrm{Bal} 31$ decreases the molecular size distribution of TTAGG fragments. The absence of a banded distribution in the smears suggest that interstitial telomeric repeats were not present and that chromosome strands were intact. Taken

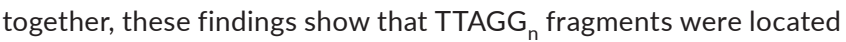
at the chromosome-ends

agreement with a recent FISH study in two other cricket species (Palacios-Gimenez et al., 2015). Together these findings support our assumption that our telomere length measurements reflect telomeric chromosome-ends.

Telomeres were on average $38.394 \mathrm{~kb}$ long (SD \pm 3.79 ), which is approximately four times longer that the telomere length of human newborns (Factor-Litvak et al., 2016), but in the same range as various other vertebrates (e.g., Gomes et al., 2011). The amongindividual coefficient of variation was $9.9 \%$ and telomere length ranged from 22-54 kb. 


\subsection{Experimental results}

Temperature treatment more than tripled the growth rate between day 0 and day 65 (Figure 3a). On day 65 , nymphs in the $23^{\circ} \mathrm{C}$ group were on average $0.12 \pm 0.01$ (SE) grams whilst nymphs in the $28^{\circ} \mathrm{C}$ group were $0.41 \pm 0.01 \mathrm{~g}$ (Table 2). Nymphs in the $23^{\circ} \mathrm{C}$ group required another 60 days to almost catch up at $0.35 \pm 0.02 \mathrm{~g} \mathrm{com}$ pared with the $28^{\circ} \mathrm{C}$ group at 65 days (Table 2). Nymphs from the intermediate age group of 85 days at $28^{\circ} \mathrm{C}$ were the heaviest at $0.49 \pm 0.01 \mathrm{~g}$.

Despite the strong effect on growth, temperature treatment did not significantly affect telomere length (Table 2; Figure 3b). All four experimental groups showed indistinguishable mean telomere lengths, indicating that neither temperature, nor chronological age had a significant effect on telomere length. Concomitantly, the overall correlation between body mass and telomere length was weak and nonsignificant ( $r=-0.034 ; p=.77)$, and there was no evidence for an interaction between temperature and age, affecting telomere length. We also estimated the effects of average family mass and delta mass (i.e., the individual deviation from the family average) on telomere length, but neither of these mass components were significant (average mass $=-16.5 \pm 9.9 \mathrm{~kb} / \mathrm{g}$; delta mass $=-2.5 \pm 2.1 \mathrm{~kb} / \mathrm{g}$; Figure $3 \mathrm{c}$ ). The slope of age versus telomere length analysed as continuous variable was 22 bp/day $(95 \%$ $\mathrm{Cl}-5.67-+50 \mathrm{bp} /$ day).

We could not detect an effect of parental altitude on the telomere length of nymphs: Neither parental altitude $(p=.409)$, nor the interaction of parental altitude $\mathrm{x}$ temperature treatment ( $p=.300)$, affected nymph telomere length (Table S1). In line with this finding, the random effect of population identity explained a negligible portion of telomere length variation (Table S1). Population was therefore excluded from further analyses due to model convergence issues inherent to random factors with small variance.

\subsection{Heritability of telomere length and body mass}

The random effect of family ID explained $64 \%$ of the total telomere length variation. We estimated this ratio based on a model that only included family ID as random effect and gel ID as fixed effect and the ratio was determined by dividing the family variance with the total variance conditional on the gel effect (i.e., family + residual). This proportion was essentially unaltered by conditioning on the fixed effects of temperature and age (Table 2 ) reflecting the fact that these effects explain negligible variance. The among family variance was remarkably significant $(p<.001)$ and indicative of high repeatability of telomere length among full siblings. Graphical presentation of the data illustrates the strong correlation of telomere length among full siblings (Figure 4). This is an important observation, first because the high repeatability implies that our null findings with respect to temperature treatment and age were unlikely to be due to low measurement accuracy. Furthermore, the high repeatability on the level of full siblings potentially reflects high heritability. Following Falconer and Mackay (1996) we derived the broad sense heritability from the family mixed effects model by multiplying the full sibling repeatability by 2 . Thus, the broad sense heritability of telomere length was 1.27 (95\% Cl 0.86-1.67). Applying the same calculations to body mass resulted in a broad sense heritability of $0.094(-0.01-0.20)$ - or 0.14 (0.02-0.27), or $0.28(0.11-0.45)$ conditional on including age or both age and temperature, respectively; the substantially lower unconditional heritability arises from the strong effects of temperature and age that contribute to phenotypic variance in body mass.

\subsection{Post hoc quantitative genetic analyses}

Such a high heritability of telomere length intuitively suggests potential for rapid evolutionary change in response to selection. However, since heritability is a ratio, i.e., genetic variation relative to the total
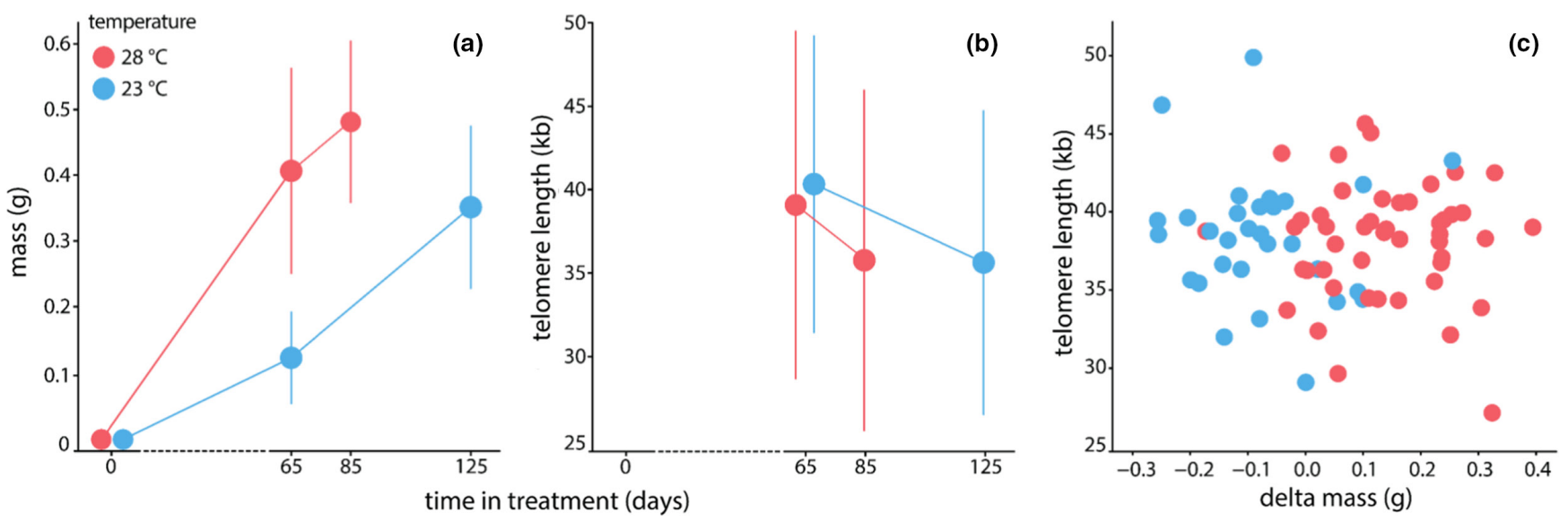

FIGURE 3 Body mass (a) and telomere length (b) of nymphs after 65-, 85-, and 125 days of temperature treatment. Nymphs started treatment when their body mass was virtually zero (included in the figure to graphically virtualise growth trajectories). Vertical bars are standard deviations. Delta mass (c) represents the individual deviation from the average mass of siblings 
TABLE 2 Body mass and telomere length in relation to temperature treatment and age

\begin{tabular}{|c|c|c|c|c|c|c|c|}
\hline Variable & $n$ & Random e & ects & $\begin{array}{l}\text { Fixed } \\
\text { effects }\end{array}$ & Estimate & SE & $p$-value \\
\hline \multirow[t]{3}{*}{ Mass } & \multirow{3}{*}{$545_{\text {nymphs }} / 59_{\text {families }}$} & Family ID & 0.0016 & $23^{\circ} \mathrm{C}$ & 0.124 & 0.009 & intercept \\
\hline & & & & day $_{-85}$ & 0.078 & 0.012 & $<.001$ \\
\hline & & & & day $_{-125}$ & 0.223 & 0.016 & $<.001$ \\
\hline \multirow{5}{*}{ Telomere length } & \multirow{5}{*}{$77_{\text {nymphs }} / 29_{\text {families }}$} & Residual & 5521789 & $28^{\circ} \mathrm{C}$ & -227 & 740 & .760 \\
\hline & & & & day $_{-85}$ & -544 & 773 & .379 \\
\hline & & & & day $_{-125}$ & -1274 & 996 & \\
\hline & & & & GellD_2 & 19046 & 1559 & $<.001$ \\
\hline & & & & GellD_3 & 19106 & 1747 & \\
\hline
\end{tabular}

Note: "n" reflects sample sizes at the individual and family levels. "random effects" shows the estimated family and residual variance components and "fixed effects" show the effects of temperature and time in treatment in days, which were included in the models as fixed factors. Note that the intercepts reflect body mass and telomere length at $23^{\circ} \mathrm{C}$ and 65 days old.

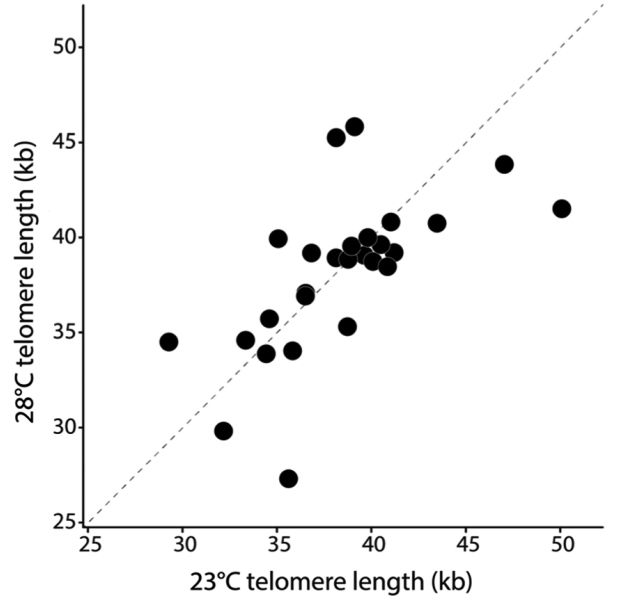

FIG URE 4 Telomere length of nymphs from the $23^{\circ} \mathrm{C}$ treatment group plotted against their siblings from the $28^{\circ} \mathrm{C}$ treatment group. The dashed line shows the line of equal values on the $y$ - and $x-$ axes. Note that deviation from the $y=x$ line is expected even when the heritability is $100 \%$ since the genetic relatedness of full siblings is 0.5 on average

variation, this is not necessarily true. We therefore conducted additional post hoc quantitative genetic analyses to directly estimate the additive genetic variance and evolvability (Houle, 1992) of telomere length. We acknowledge that our study design is not optimal for this as the absence of any half-sibling structure means we cannot statistically separate additive genetic variance from any parental and/ or dominance variance present. Noting that these additional posthoc results should thus be interpreted with caution, we fitted univariate animal models of telomere length and body mass to partition additive genetic and residual (environmental) variance components. Scaling to broad sense (or narrow sense if we assume an absence of parental and dominance variance) heritabilities corroborated our family-model estimates. For telomere length, heritability was $\sim 1$ with a standard error $<0.0001$ and the estimate was unaffected when conditioning the estimate on fixed effects of age and/or treatment. For body mass, we estimated a heritability $(95 \% \mathrm{Cl})$ of $0.094(-0.01-$ $0.198)$, increasing to $0.145(0.0196-0.270)$ and 0.279 (0.108-0.450) when age, or both age and temperature were included in the model as fixed effects.

We used the estimated (additive) genetic variance to calculate the coefficient of genetic variation: $C V_{A}(x)=\sqrt{V_{A}} / \bar{x}$, where $V_{A}$ is the (assumed additive) genetic variance of telomere length $(x)$ that is estimated by the pedigree random effect. Following Houle (1992) and Garcia-Gonzalez et al., (2012), the evolvability is equal to the square of $C V_{A}$ and ranges from $0 \%-100 \%$. The telomere length coefficient of genetic variation was low (9.33\%) resulting in a low evolvability of $0.87 \%$. In comparison, the body mass coefficient of genetic variation was higher (19.06\%) resulting in a higher evolvability of $3.64 \%$, despite the lower broad sense heritability of $9.4 \%$. Notably, the relatively low genetic variation on the mean standardised scale of telomere length is likely to limit its potential for evolutionary change under selection despite the high heritability.

Finally, we ran a bivariate animal model to determine the extent of genetic variation among genotypes in the response to temperature treatment (i.e., GxE). We did this to check the possibility of a GxE interaction resulting in the absence of an overall effect of temperature on telomere length (Figure $3 \mathrm{~b}$ ). This model estimates the additive genetic variance within each temperature condition $\left(V_{A 23}\right.$ and $\left.V_{A 28}\right)$ and the genetic covariance $\left(\operatorname{cov}_{A}\right)$ among them from which the genetic correlation can be derived: $r_{G}=\frac{\operatorname{cov}_{A}}{\sqrt{V_{A 23} \cdot V_{A 28}}} . G \times E$ interactions are observed either when $r_{G}$ is significantly below 1 , and/ or when the two additive genetic variances are significantly different. Age was included in both models as a fixed factor such that the observed genetic heterogeneity is over and above the effects of age. Gel ID was included as additional fixed factor for the telomere length model. We found that the genetic correlations $( \pm \mathrm{SE})$ did not significantly deviate from 1 for body mass $\left(r_{G}=0.62 \pm 0.27\right.$; 
$p=.133)$ or telomere length $\left(r_{G}=\sim 1 \pm 0.001 ; p=.99\right.$; Tables S2S3). However, for body mass $V_{A 23}$ was significantly smaller than $V_{A 28}$ $\left(V_{A 23}=0.0021 \pm 0.0011 ; V_{A 28}=0.0056 \pm 0.0021 ; p=<.031\right)$ supporting GxE (Table S3). Graphical inspection of the data agreed with these findings (Figure 5).

In conclusion, our analyses do not support the hypothesis that temperature induced growth acceleration is achieved at the cost of telomere length in field crickets. However, in considering the implications of this finding (below) it is worth noting that the absolute mean telomere length difference between the two temperatures was 227 bp after 65 days of treatment (Table 2) which is substantial on an absolute scale when compared with previous studies that manipulated growth, e.g., Boonekamp et al., (2014). Likewise, the rate of telomere attrition was 22 base pairs per day, which approximately equals the annual telomere loss in adult humans. In this context it is important to recognize that the size of the "effect" depends on what variance scaling is used and it remains unclear which scaling is the most informative with respect to fitness: For example, the effect size may be expressed as the mean difference between experimental groups relative to a common standard deviation, i.e., Cohen's $d$
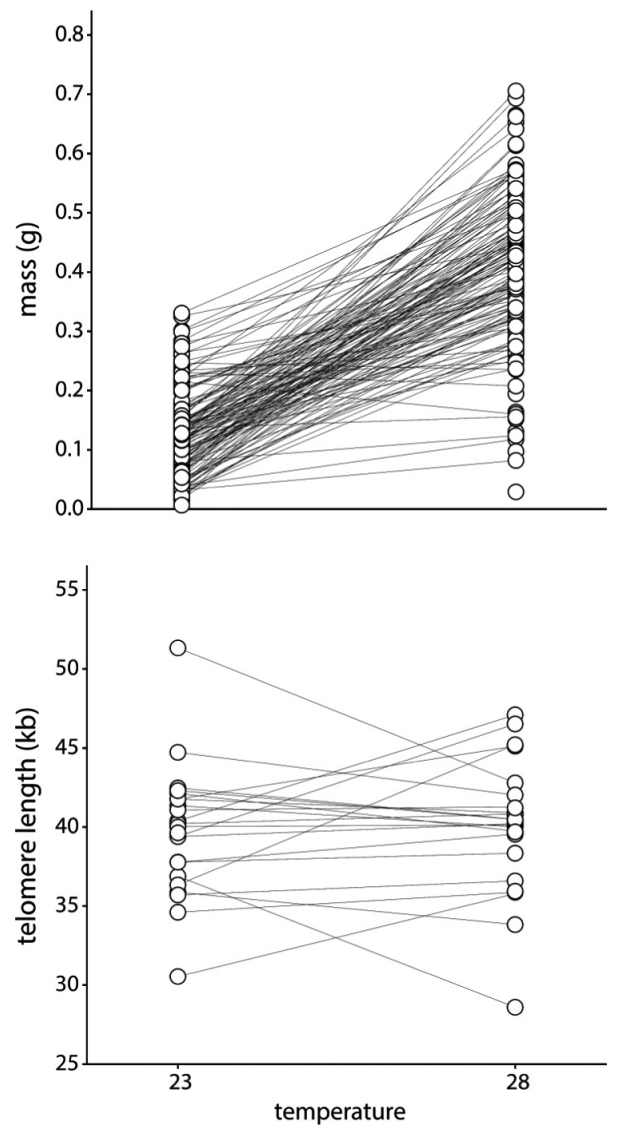

FIGURE 5 Body mass and telomere length in response to temperature treatment. Lines connect siblings. Note that for body mass, this figure only shows the 65 days after treatment subset because there were strong effects of age (in the statistical analyses we included all data and controlled for age). All telomere data was included in this figure since the effect of age on telomere length was not statistically significant
(Nakagawa \& Cuthill, 2007). Given the high heritability it may also be useful to express the mean difference among treatments relative to the family residual variance thus expressing the effect of temperature on telomere length relative to variation within families. The first estimate results in a very low effect size of the temperature treatment (Cohen's $d=0.06$ ), whereas the latter approach results in a higher (albeit still small [Cohen., 2013]) effect size of $d=0.12$. It remains to be tested which scaling is more predictive of fitness and it could be that scaling to the family level variance could be more informative when this approach yields a better estimate of the rate of telomere attrition. A detailed study in the wild linking telomere length and the rate of telomere attrition to fitness is required to further our understanding of these variance scaling issues.

\section{DISCUSSION}

Telomere dynamics have recently been proposed as a mechanism linking growth to fitness in vertebrates, for example Monaghan and Ozanne (2018). Metabolic rate and oxidative stress have been shown to increase with temperature in some insect species (Das et al., 2018; Lalouette et al., 2011) and, based on this, we expected growth acceleration by temperature to shorten telomeres, possibly through higher oxidative stress. However, we found that temperature-induced acceleration of growth had no discernible effects on telomere length near the end of the experimental growth period in field crickets.

Our findings are in contrast to a comparable study showing that warmer temperatures accelerated prehatching growth and reduced telomere length in a bird species, the common tern (Vedder et al., 2018). A notable difference between the two study species is that crickets are ectotherms, and as such differ in their physiological response to environmental temperature from that of endotherms. On the other hand, bird embryos are strictly speaking ectothermic during their early development, although their range of temperature tolerance is probably more limited, and the role of ectothermy therefore remains speculative. It is interesting in this context that a study of another ectotherm, the Atlantic salmon (Salmo salar), also found no effect of temperature induced growth rate variation on telomere length (McLennan et al., 2018). These findings are unexpected because even if growth acceleration does not incur any fitness cost - as may be the case when growth is entirely temperature dependent we would still expect the absolute number of cell divisions to affect telomere length. This raises the question of the importance of mechanisms that actively maintain telomere length irrespective of age, size and growth rate, for example through the activity of telomerase (Collins \& Mitchell, 2002). From an evolutionary perspective, the answer to this question depends upon the level of additive genetic variation: (a) High additive genetic variation may exist because it accumulated in the absence of selection on the trait. This is the default hypothesis, and it assumes that the observed phenotypic variation is neutral with respect to selection. (b) Low additive genetic variation on the other hand may indicate strong environmental canalisation of 
the trait, which could reflect an adaptation (Boonekamp et al., 2017, 2018; Stearns \& Kawecki, 1994; Vedder et al., 2017; Waddington, 1942). Below, we discuss these two possibilities.

1. A common explanation for high genetic variation in a trait is that selection is weak allowing variation to accumulate. This is particularly easy to envisage in relation to telomere length because it represents non-coding DNA. Indeed, recent reviews of the literature have found that telomere length is highly heritable across vertebrates (Atema et al., 2015; Dugdale \& Richardson, 2018), including humans (e.g., Broer et al., 2013) and our observation fits this pattern. However, it remains unclear to what extent these empirical estimates of telomere length heritability reflect the amount of additive genetic variance, or alternatively, that high heritability reflects low environmental variance (Garcia-Gonzalez et al., 2012; Hansen et al., 2011). Hence, interpretability is enhanced by providing estimates of additive genetic variance alongside estimates of heritability (Morrissey et al., 2010).

In addition to scaling the additive genetic variance to the residual variance (heritability), we used scaling to the mean of telomere length $\left(C V_{A}\right)$. The advantage of expressing the additive genetic variance as a coefficient of variation, is that it facilitates comparison of levels of genetic variation among traits and species with phenotypic values on different absolute scales and hence has long been the recommended technique in evolutionary studies (Houle, 1992). Note however, that scaling to the mean may only be useful when the telomere assay method yields a quantitative scale that is informative with respect to the absolute telomere length measured in base pairs.

We found that the $C V_{A}$ of body mass was about double the $C V_{A}$ of telomere length, despite the much higher heritability of telomere length. The relatively low $C V_{A}$ of telomere length is unlikely to reflect selective sampling because we sampled from 10 discrete, geographically isolated populations (Table 1) and there was no effect of altitude on telomere length (Table S1). Our findings are in agreement with two similar analyses recently conducted in wild jackdaw nestlings (Bauch et al., 2020) and adult common terns (Vedder et al., 2021), which also showed high heritability of telomere length together with a low $C V_{A}$. We are unaware of any other (non-qPCR) telomere length studies reporting $C V_{A}$ (or $V_{A}$ ) and it remains to be seen therefore to what extent our findings can be generalised. Nevertheless, these three studies highlight the importance of scaling of the additive genetic variance because the two different scaling methods yield opposing interpretations regarding telomere length evolution: The high heritability suggests the potential of strong evolutionary change in the presence of selection, whereas the low $C V_{A}$ reflects a low amount of additive genetic variance, relative to the trait mean, limiting evolvability. Unfortunately, the evolutionary dynamics of telomere length will remain elusive until direct estimates of the additive genetic covariance between telomere length and fitness become available. Vedder et al., (2021) estimated the additive genetic covariance between telomere length and lifespan in the common tern, revealing a modest genetic correlation $\left(r_{G}=0.39\right)$ but with the confidence interval overlapping with zero. More studies like these will be required to improve our understanding of the extent to which telomere length causally underpins individual fitness variation.

2. If telomere length is a selectively neutral trait, we might expect to see greater levels of genetic variation than we observed for body mass, which we assume not to be selectively neutral as supported by a previous study of insects (Waller \& Svensson, 2017). Our finding of less genetic variation for telomere length suggests it is robust and maintained at its current level, consistent with adaptive environmental canalisation. The plausibility of this hypothesis depends on the assumptions (a) that telomere length causally affects fitness and (b) that there are physiological mechanisms that maintain telomere length in the face of environmental perturbations:

a. Telomere length is predictive of survival in vertebrates, overall showing a $20 \%$ increased mortality risk per standard deviation of lower telomere length (Wilbourn et al., 2018). Whether the same is true in insects remains to be verified. We have demonstrated the existence of both actuarial and phenotypic senescence in the adult phase of the life cycle of our wild field crickets (Rodríguez-Muñoz, Boonekamp, Liu, Skicko, Haughland Pedersen, et al., 2019), and similar observations have been made in other insect species (Bonduriansky \& Brassil, 2002; Hunt et al., 2004; Sherratt et al., 2011; Tatar et al.,1993). We therefore expect that, similar to vertebrate species, accurate markers of physiological senescence should predict the remaining adult lifespan of insects. G. campestris spend at least $70 \%$ of their life as growing nymphs, and we anticipated that this extensive period of growth / cell divisions should be accompanied with substantial telomere attrition. The absence of an effect of manipulated growth on telomere attrition and the low level of genetic variation suggests that telomere length is strongly maintained, consistent with the canalisation hypothesis. It is therefore of great interest to investigate potential mechanisms that could support the canalisation of telomere length.

b. Recent studies have identified potential canalisation mechanisms for telomere length in vertebrates. The combined action of sister-chromatid exchange and colocalization of the DNA recombination proteins Rad50 and TRF1 has been found to cause telomere elongation during early embryogenesis (Liu et al., 2007). The observed genetic variation of telomere length therefore depends on the inheritance of such telomere elongation mechanisms during early embryogenesis, over and above the inherited initial telomere length of parental gametes. Furthermore, another study shows that telomeres that become too long during early development are trimmed back by telomeric zinc finger-associated protein expression ( $\mathrm{Li}$ et al., 2017). These studies show that mechanisms are in place that elongate and shorten 
chromosome-ends during early development to create telomeres of a certain length. The high heritability of telomere length suggests that the joint action of these mechanisms is heritable, resulting in a canalised, developmentally robust predetermined telomere length. At the same time, epigenetic effects have also been observed showing that offspring telomere length decreases with paternal age (for a longitudinal study see Bauch et al., 2019). The presence of such epigenetic effects implies that telomere length regulatory mechanisms may be limited in their capacity to fully compensate for the telomere length that was physically inherited from the paternal gametes. It will be fascinating to investigate in detail whether canalisation of telomere length occurs during early development, followed by subsequent decanalisation during growth and ageing, and their impact on individual variation. A recent study shows supporting evidence for this pattern: incubation temperature had no effect on the initial telomere length of quail embryos, but temperature altered growth rate and increased the heterogeneity of telomere length post-hatching, reminiscent of decanalisation (Stier et al., 2020).

Functional explanations for a causal relationship between telomere dynamics and fitness are based on the premise that telomeres lose their protective function inducing cell cycle arrest when they erode to a critical limit. We found that the rate of telomere attrition was $22 \mathrm{bp} /$ day, which amounts to a daily loss rate of $0.06 \%$. Although we do not know what the critical telomere length is in field crickets, this slope implies that telomeres of an average of $38 \mathrm{~kb}$ would be fully eroded after 4.5 years (or two full years if we take the upper $95 \%$ confidence limit of the slope of age). This suggests that it is unlikely that there is a causal effect of telomere length limiting lifespan in this annual insect unless there are bouts of substantially accelerated telomere attrition during a later life stage. On the other hand, it should be noted that we used the average telomere length of a distribution of different telomere lengths within a sample/individual. This within-sample variation stems from heterogeneity among cells, lineages, and even chromosomes with differing telomere lengths within cells. The argument has been made that the chromosomes with the shortest telomeres cause senescence (Baird et al., 2003; Zou et al., 2004). The TRF method we used allowed us to look at the distribution of telomere lengths within a sample. The shortest telomeres we could detect (i.e., the $5 \%$ percentile) ranged from $2.7 \mathrm{~kb}$ to $24.5 \mathrm{~kb}$ (average $\pm S D$ : $15 \pm 5.6 \mathrm{~kb}$ ). This range suggests that for some individuals the shortest telomeres could easily be fully eroded within a year. Our findings therefore neither support nor refute a possible causal limit of telomere dynamics on the lifespan of this species.

Field crickets are an ancient species dating back to the Triassic (Song et al., 2015). Our observation that field crickets have their chromosome-ends capped by telomeres, just like vertebrate species, illustrates the strong evolutionary conservation of these chromosomal structures. It also raises the question of whether there are fundamental similarities or differences in telomere function between vertebrates and other groups. Recent syntheses propose that telomere length of vertebrates may be under stabilizing selection by functioning as an anti-cancer mechanism (Aviv et al., 2017; Young, 2018). This hypothesis is primarily based on the observation that larger vertebrates with long periods of growth tend to have shorter telomeres than small vertebrates and the premise that this relationship coexists with a higher cancer risk in larger species (Gomes et al., 2011). Shorter telomere length could be adaptive in large vertebrates when it selectively shuts down cell proliferation of unstable cells. Insects on the other hand have been suggested to be virtually free from cancer (Scharrer \& Lochhead, 1950) and this could be the basis for a fundamental difference between vertebrate and invertebrate groups in the link between growth and telomere dynamics. Consistent with this hypothesis we found that field crickets show surprisingly long telomeres of $\sim 38 \mathrm{~kb}$, much longer than many species of birds and mammals. However, detailed information on the occurrence of cancer is scant for wild insects and tumour growth has been documented in dung beetles (Scharrer \& Lochhead, 1950), which live much longer than most insects. Clearly, more studies are required on different insects and other invertebrates to determine systematic similarities and/or differences of telomere function among taxonomic groups.

\section{ACKNOWLEDGEMENTS}

Caleb Peters, Emma May, Paola Leone and Zinnia Pennington collected most of the wild adult crickets used in this study. Anne Meurs aided with cricket husbandry, body mass measurements and snap-freezing of nymphs. This work was supported by the Natural Environment Research Council (NERC) standard grant NE/ R000328/1 and the European Union's Horizon 2020 research and innovation programme under the Marie Skłodowska-Curie grant agreement No 792215 (Boonekamp).

\section{AUTHOR CONTRIBUTIONS}

This study was designed by Jelle Boonekamp, Simon Verhulst, and Tom Tregenza. Adults were mated and eggs were collected and incubated by Rolando Rodríguez-Muñoz and Paul Hopwood. Jelle Boonekamp conducted the temperature manipulation experiment in Groningen. Erica Zuidersma and Ellis Mulder snap froze nymphs, measured body mass, and carried out the DNA extractions and telomere length analyses. $\mathrm{Bal} 31$ assays were done by Jelle Boonekamp and Ellis Mulder. Statistical analyses were performed by Jelle Boonekamp with help from Alastair Wilson, Simon Verhulst and Tom Tregenza. Jelle Boonekamp wrote the first draft and all authors contributed to the final version.

\section{DATA AVAILABILITY STATEMENT}

Data is available from the Dryad Digital Repository: https://doi. org/10.5061/dryad.jdfn2z39z. https://doi.org/10.5061/dryad.jdfn2 $z 39 z$ 


\section{ORCID}

Jelle Boonekamp (1) https://orcid.org/0000-0003-1900-627X

Rolando Rodríguez-Muñoz (1) https://orcid.org/0000-0002-6067-845X

Paul Hopwood (1) https://orcid.org/0000-0003-1511-3705

Erica Zuidersma (10) https://orcid.org/0000-0001-6138-080X

Ellis Mulder (1) https://orcid.org/0000-0002-1956-8734

Alastair Wilson (10 https://orcid.org/0000-0002-5045-2051

Simon Verhulst (1) https://orcid.org/0000-0002-1143-6868

Tom Tregenza (1) https://orcid.org/0000-0003-4182-2222

\section{REFERENCES}

Alonso-Alvarez, C., Bertrand, S., Faivre, B., \& Sorci, G. (2007). Increased susceptibility to oxidative damage as a cost of accelerated somatic growth in zebra finches. Functional Ecology, 21, 873-879.

Armanios, M., \& Blackburn, E. H. (2012). The telomere syndromes. Nature Reviews Genetics, 13, 693-704.

Atema, E., Mulder, E., Dugdale, H. L., Briga, M., van Noordwijk, A. J., \& Verhulst, S. (2015). Heritability of telomere length in the Zebra Finch. Journal of Ornithology, 156, 1113-1123.

Atema, E., Mulder, E., van Noordwijk, A. J., \& Verhulst, S. (2019). Ultralong telomeres shorten with age in nestling great tits but are static in adults and mask attrition of short telomeres. Molecular Ecology Resources, 19, 648-658.

Aviv, A., Anderson, J. J., \& Shay, J. W. (2017). Mutations, cancer and the telomere length paradox. Trends in Cancer, 3, 253-258.

Baird, D. M., Rowson, J., Wynford-Thomas, D., \& Kipling, D. (2003). Extensive allelic variation and ultrashort telomeres in senescent human cells. Nature Genetics, 33, 203-207.

Bauch, C., Boonekamp, J. J., Korsten, P., Mulder, E., \& Verhulst, S. (2019). Epigenetic inheritance of telomere length in wild birds. Plos Genetics, 15, e1007827.

Bauch, C., Boonekamp, J. J., Korsten, P., Mulder, E., \& Verhulst, S. (2020). High heritability of telomere length, but low evolvability, and no significant heritability of telomere shortening in wild jackdaws. BioRxiv, 2020.12.16.423128.

Benetos, A., Verhulst, S., Labat, C., Lai, T. P., Girerd, N., Toupance, S., Zannad, F., Rossignol, P., \& Aviv, A. (2019). Telomere length tracking in children and their parents: implications for adult onset diseases. The FASEB Journal, 33, 14248-14253.

Blackburn, E. H. (1991). Structure and function of telomeres. Nature, 350, 569-573.

Bonduriansky, R., \& Brassil, C. E. (2002). Senescence: Rapid and costly ageing in wild male flies. Nature, 420, 377.

Boonekamp, J. J., Dijkstra, R., Dijkstra, C., \& Verhulst, S. (2017). Canalization of development reduces the utility of traits as fitness biomarkers: Feather fault bars in nestling birds. Functional Ecology, 31, 719-727.

Boonekamp, J. J., Mulder, E., Salomons, H. M., Dijkstra, C., \& Verhulst, S. (2014). Nestling telomere shortening, but not telomere length, reflects developmental stress and predicts survival in wild birds. Proceedings of the Royal Society of London Series B-Biological Sciences, 281, 20133287.

Boonekamp, J. J., Mulder, E., \& Verhulst, S. (2018). Canalisation in the wild: effects of developmental conditions on physiological traits are inversely linked to their association with fitness. Ecology Letters, $67,1$.

Boonekamp, J. J., Simons, M. J. P., Hemerik, L., \& Verhulst, S. (2013). Telomere length behaves as biomarker of somatic redundancy rather than biological age. Aging Cell, 12, 330-332.

Broer, L., Codd, V., Nyholt, D. R., Deelen, J., Mangino, M., Willemsen, G., Albrecht, E., Amin, N., Beekman, M., De Geus, E. J., \& Henders, A. (2013). Meta-analysis of telomere length in 19,713 subjects reveals high heritability, stronger maternal inheritance and a paternal age effect. European Journal of Human Genetics, 21, 1163-1168.

Butler, D. G., Cullis, B. R., Gilmour, A. R., Gogel, B. J., \& Thompson, R. (2018). ASReml-R reference manual version 4. VSN International Ltd.

Calado, R. T., \& Young, N. S. (2009). Telomere diseases. New England Journal of Medicine, 361, 2353-2365.

Chatelain, M., Drobniak, S. M., \& Szulkin, M. (2019). The association between stressors and telomeres in non-human vertebrates: A metaanalysis. Ecology Letters, 99, 21.

Cohen, J. (2013). Statistical power analysis for the behavioral sciences. Routledge.

Collins, K., \& Mitchell, J. R. (2002). Telomerase in the human organism. Oncogene, 21, 564-579.

Das, S., Malik, U., \& Barik, A. (2018). Effect of thermal stress on antioxidant responses of the biocontrol agent Galerucella placida (Coleoptera: Chrysomelidae). International Journal of Tropical Insect Science, 38, 400-409.

Dmitriew, C. M. (2011). The evolution of growth trajectories: What limits growth rate? Biological Reviews, 86, 97-116.

Dugdale, H. L., \& Richardson, D. S. (2018). Heritability of telomere variation: It is all about the environment!. Philosophical Transactions of the Royal Society B: Biological Sciences, 373, 20160450.

Eastwood, J. R., Hall, M. L., Teunissen, N., Kingma, S. A., Hidalgo Aranzamendi, N., Fan, M., Roast, M., Verhulst, S., \& Peters, A. (2019). Early-life telomere length predicts lifespan and lifetime reproductive success in a wild bird. Molecular Ecology, 28, 1127-1137.

Entringer, S., Epel, E. S., Kumsta, R., Lin, J., Hellhammer, D. H., Blackburn, E. H., Wust, S., \& Wadhwa, P. D. (2011). Stress exposure in intrauterine life is associated with shorter telomere length in young adulthood. Proceedings of the National Academy of Sciences, 108, E513-E518.

Epel, E. S., Blackburn, E. H., Lin, J., Dhabhar, F. S., Adler, N. E., Morrow, J. D., \& Cawthon, R. M. (2004). Accelerated telomere shortening in response to life stress. Proceedings of the National Academy of Sciences, 101, 17312-17315.

Factor-Litvak, P., Susser, E., Kezios, K., McKeague, I., Kark, J. D., Hoffman, M., Kimura, M., Wapner, R., \& Aviv, A. (2016). Leukocyte telomere length in newborns: Implications for the role of telomeres in human disease. Pediatrics, 137, e20153927.

Falconer, D. S., \& Mackay, T. (1996). Introduction to quantitative genetics. Longman.

Frangakis, C. E., \& Rubin, D. B. (2002). Principal stratification in causal inference. Biometrics, 58, 21-29.

Frydrychová, R., Grossmann, P., Trubac, P., Vítková, M., \& Marec, F. E. (2004). Phylogenetic distribution of TTAGG telomeric repeats in insects. Genome, 47, 163-178.

Garcia-Gonzalez, F., Simmons, L. W., Tomkins, J. L., Kotiaho, J. S., \& Evans, J. P. (2012). Comparing evolvabilities: Common errors surrounding the calculation and use of coefficients of additive genetic variation. Evolution, 66, 2341-2349.

Gilbert, N., \& Raworth, D. A. (1996). Forum: Insects and temperature-a general theory. The Canadian Entomologist, 128, 1-13.

Gomes, N. M. V., Ryder, O. A., Houck, M. L., Charter, S. J., Walker, W., Forsyth, N. R., Austad, S. N., Venditti, C., Pagel, M., Shay, J. W., \& Wright, W. E. (2011). Comparative biology of mammalian telomeres: Hypotheses on ancestral states and the roles of telomeres in longevity determination. Aging Cell, 10, 761-768.

Gomes, N. M. V., Shay, J. W., \& Wright, W. E. (2010). Telomere biology in Metazoa. FEBS Letters, 584, 3741-3751.

Hansen, T. F., Pélabon, C., \& Houle, D. (2011). Heritability is not evolvability. Evolutionary Biology, 38, 258-277.

Heidinger, B. J., Blount, J. D., Boner, W., Griffiths, K., Metcalfe, N. B., \& Monaghan, P. (2012). Telomere length in early life predicts lifespan. Proceedings of the National Academy of Sciences, 109, 1743-1748. 
Houle, D. (1992). Comparing evolvability and variability of quantitative traits. Genetics, 130, 195-204.

Hug, N., \& Lingner, J. (2006). Telomere length homeostasis. Chromosoma, $115,413-425$

Hunt, J., Brooks, R., Jennions, M. D., Smith, M. J., Bentsen, C. L., \& Bussière, L. F. (2004). High-quality male field crickets invest heavily in sexual display but die young. Nature, 432, 1024-1027.

Jemielity, S., Kimura, M., Parker, K. M., Parker, J. D., Cao, X., Aviv, A., \& Keller, L. (2007). Short telomeres in short-lived males: what are the molecular and evolutionary causes? Aging Cell, 6, 225-233.

Korandová, M., Krůček, T., Vrbová, K., \& Frydrychova, R. C. (2014). Distribution of TTAGG-specific telomerase activity in insects. Chromosome Research, 22, 495-503.

Lalouette, L., Williams, C. M., Hervant, F., Sinclair, B. J., \& Renault, D. (2011). Metabolic rate and oxidative stress in insects exposed to low temperature thermal fluctuations. Comparative Biochemistry and Physiology a-Molecular \& Integrative Physiology, 158, 229-234.

Li, J. S. Z., Miralles Fusté, J., Simavorian, T., Bartocci, C., Tsai, J., Karlseder, J., \& Lazzerini Denchi, E. (2017). TZAP: A telomereassociated protein involved in telomere length control. Science, 355, 638-641.

Lin, J., Epel, E., \& Blackburn, E. H. (2012). Telomeres and lifestyle factors: Roles in cellular aging. Mutation Research/Fundamental and Molecular Mechanisms of Mutagenesis, 730, 85-89.

Liu, L., Bailey, S. M., Okuka, M., Muñoz, P., Li, C., Zhou, L., Wu, C., Czerwiec, E., Sandler, L., Seyfang, A., Blasco, M. A., \& Keefe, D. L. (2007). Telomere lengthening early in development. Nature Cell Biology, 9, 1436-1441.

McLennan, D., Armstrong, J. D., Stewart, D. C., Mckelvey, S., Boner, W., Monaghan, P., \& Metcalfe, N. B. (2018). Telomere elongation during early development is independent of environmental temperatures in Atlantic salmon. Journal of Experimental Biology, 221, jeb178616.

Monaghan, P., \& Ozanne, S. E. (2018). Somatic growth and telomere dynamics in vertebrates: relationships, mechanisms and consequences. Philosophical Transactions of the Royal Society B: Biological Sciences, 373, 20160446.

Morrissey, M. B., Kruuk, L. E. B., \& Wilson, A. J. (2010). The danger of applying the breeder's equation in observational studies of natural populations. Journal of Evolutionary Biology, 23, 2277-2288.

Nakagawa, S., \& Cuthill, I. C. (2007). Effect size, confidence interval and statistical significance: A practical guide for biologists. Biological Reviews, 82, 591-605.

Okazaki, S., Tsuchida, K., Maekawa, H., Ishikawa, H., \& Fujiwara, H. (1993). Identification of a pentanucleotide telomeric sequence, (TTAGG)n, in the silkworm Bombyx mori and in other insects. Molecular and Cellular Biology, 13, 1424-1432.

Olovnikov, A. M. (1973). A theory of marginotomy. The incomplete copying of template margin in enzymic synthesis of polynucleotides and biological significance of the phenomenon. Journal of Theoretical Biology, 41, 181-190.

Palacios-Gimenez, O. M., Carvalho, C. R., Ferrari Soares, F. A., \& Cabralde-Mello, D. C. (2015). Contrasting the chromosomal organization of repetitive DNAs in two Gryllidae crickets with highly divergent karyotypes. PLoS One, 10, e0143540.

R Core Team (2020). R: A language and environment for statistical computing. R Foundation for Statistical Computing.

Richard, G., Le Trionnaire, G., Danchin, É., \& Sentis, A. (2019). Epigenetics and insect polyphenism: Mechanisms and climate change impacts. Current Opinion in Insect Science, 38, 138-145.

Rodríguez-Muñoz, R., Boonekamp, J. J., Liu, X. P., Skicko, I., Fisher, D. N., Hopwood, P., \& Tregenza, T. (2019). Testing the effect of earlylife reproductive effort on age-related decline in a wild insect. Evolution, 73, 317-328.

Rodríguez-Muñoz, R., Boonekamp, J. J., Liu, X. P., Skicko, I., Haughland Pedersen, S., Fisher, D. N., Hopwood, P., \& Tregenza, T. (2019).
Comparing individual and population measures of senescence across 10 years in a wild insect population. Evolution, 73, 293-302.

Roe, R. M., Clifford, C. W., \& Woodring, J. P. (1980). The effect of temperature on feeding, growth, and metabolism during the last larval stadium of the female house cricket, Acheta domesticus. Journal of Insect Physiology, 26, 639-644.

Salomons, H. M., Mulder, G. A., van de Zande, L., Haussmann, M. F., Linskens, M. H. K., \& Verhulst, S. (2009). Telomere shortening and survival in free-living corvids. Proceedings of the Royal Society of London Series B-Biological Sciences, 276, 3157-3165.

Scharrer, B., \& Lochhead, M. S. (1950). Tumors in the invertebrates: A review. Cancer Research, 10, 403-419.

Sherratt, T. N. T., Hassall, C. C., Laird, R., Thompson, D. J. D., \& CorderoRivera, A. A. (2011). A comparative analysis of senescence in adult damselflies and dragonflies (Odonata). Journal of Evolutionary Biology, 24, 810-822.

Smith, S. M., Nager, R. G., \& Costantini, D. (2016). Meta-analysis indicates that oxidative stress is both a constraint on and a cost of growth. Ecology and Evolution, 6, 2833-2842.

Song, H., Amédégnato, C., Cigliano, M. M., Desutter-Grandcolas, L., Heads, S. W., Huang, Y., Otte, D., \& Whiting, M. F. (2015). 300 million years of diversification: elucidating the patterns of orthopteran evolution based on comprehensive taxon and gene sampling. Cladistics, 31, 621-651.

Stearns, S. C., \& Kawecki, T. J. (1994). Fitness sensitivity and the canalization of life-history traits. Evolution, 48, 1438.

Stewart, S., Ben-Porath, I., Carey, V., O'Connor, B. F., Hahn, W. C., \& Weinberg, R. A. (2003). Erosion of the telomeric single-strand overhang at replicative senescence. Nature Genetics, 33, 492-496.

Stier, A., Metcalfe, N. B., \& Monaghan, P. (2020). Pace and stability of embryonic development affect telomere dynamics: An experimental study in a precocial bird model. Proceedings of the Royal Society B: Biological Sciences, 287, 20201378.

Tatar, M., Carey, J. R., \& Vaupel, J. W. (1993). Long-term cost of reproduction with and without accelerated senescence in Callosobruchus maculatus: analysis of age-specific mortality. Evolution, 47, 1302-1312.

Tricola, G. M., Simons, M. J. P., Atema, E., Boughton, R. K., Brown, J. L., Dearborn, D. C., Divoky, G., Eimes, J. A., Huntington, C. E., Kitaysky, A. S., Juola, F. A., Lank, D. B., Litwa, H. P., Mulder, E. G. A., Nisbet, I. C. T., Okanoya, K., Safran, R. J., Schoech, S. J., Schreiber, E. A., ... Haussmann, M. F. (2018). The rate of telomere loss is related to maximum lifespan in birds. Philosophical Transactions of the Royal Society B: Biological Sciences, 373, 20160445.

Türkoğlu, Ş., Koca, S., \& Akpınar, N. (2003). Karyological observations on the Field Cricket, Gryllus campestris L. (Gryllidae, Orthoptera). Zoology in the Middle East, 28, 113-117.

Vedder, O., Moiron, M., Bichet, C., Bauch, C., Verhulst, S., Becker, P. H., $\&$ Bouwhuis, S. (2021). Telomere length is heritable and genetically correlated with lifespan in a wild bird. Molecular Ecology, 1-11. https://doi.org/10.1111/mec.15807

Vedder, O., Verhulst, S., Bauch, C., \& Bouwhuis, S. (2017). Telomere attrition and growth: A life-history framework and case study in common terns. Journal of Evolutionary Biology, 30, 1409-1419.

Vedder, O., Verhulst, S., Zuidersma, E., \& Bouwhuis, S. (2018). Embryonic growth rate affects telomere attrition: An experiment in a wild bird. Journal of Experimental Biology, 221, jeb181586.

Vítková, M., Král, J., Traut, W., Zrzavý, J., \& Marec, F. (2005). The evolutionary origin of insect telomeric repeats, (TTAGG)n. Chromosome Research, 13, 145-156.

von Zglinicki, T. (2002). Oxidative stress shortens telomeres. Trends in Biochemical Sciences, 27, 339-344.

Waddington, C. H. (1942). Canalization of development and the inheritance of acquired characters. Nature, 150, 563-565.

Waller, J. T., \& Svensson, E. I. (2017). Body size evolution in an old insect order: No evidence for Cope's Rule in spite of fitness benefits of large size. Evolution, 71, 2178-2193. 
Wilbourn, R. V., Moatt, J. P., Froy, H., Walling, C. A., Nussey, D. H., \& Boonekamp, J. J. (2018). The relationship between telomere length and mortality risk in non-model vertebrate systems: A metaanalysis. Philosophical Transactions of the Royal Society B: Biological Sciences, 373, 20160447.

Young, A. J. (2018). The role of telomeres in the mechanisms and evolution of life-history trade-offs and ageing. Philosophical Transactions of the Royal Society B: Biological Sciences, 373, 20160452.

Zeichner, S. L., Palumbo, P., Feng, Y. R., Xiao, X., Gee, D., Sleasman, J., Goodenow, M., Biggar, R., \& Dimitrov, D. (1999). Rapid telomere shortening in children. Blood, 93, 2824-2830.

Zou, Y., Sfeir, A., Gryaznov, S. M., Shay, J. W., \& Wright, W. E. (2004). Does a sentinel or a subset of short telomeres determine replicative senescence? Molecular Biology of the Cell, 15, 3709-3718.

\section{SUPPORTING INFORMATION}

Additional supporting information may be found online in the Supporting Information section.

How to cite this article: Boonekamp J, Rodríguez-Muñoz R, Hopwood P, et al. Telomere length is highly heritable and independent of growth rate manipulated by temperature in field crickets. Mol Ecol. 2022;31:6128-6140. https://doi. org/10.1111/mec.15888 